\title{
Dipolarization fronts in the near-Earth space and substorm dynamics
}

\author{
I. I. Vogiatzis ${ }^{1,2}$, A. Isavnin ${ }^{3}$, Q.-G. Zong ${ }^{2}$, E. T. Sarris ${ }^{1}$, S. W. Lu ${ }^{2}$, and A. M. Tian ${ }^{4}$ \\ ${ }^{1}$ Department of Electrical and Computer Engineering, Democritus University of Thrace, Xanthi, Greece \\ ${ }^{2}$ Institute of Space Physics and Applied Technology, Peking University, Beijing, China \\ ${ }^{3}$ Department of Physics, University of Helsinki, P.O. Box 64, 00014, Finland \\ ${ }^{4}$ School of Space Science and Physics, Shandong University, Weihai, China \\ Correspondence to: I. I. Vogiatzis (ivogiatz@ee.duth.gr)
}

Received: 28 July 2014 - Revised: 4 December 2014 - Accepted: 5 December 2014 - Published: 15 January 2015

\begin{abstract}
During magnetospheric substorms and plasma transport in the Earth's magnetotail various magnetic structures can be detected. Dipolarization fronts and flux ropes are the most prominent structures characteristic for substorm dynamics. However, they are treated as separate magnetotail features independent of each other. In this paper, we analyze a number of dipolarization fronts observed by the THEMIS (Time History of Events and Macroscale Interactions during Substorms) spacecraft at different geocentric distances by applying the magnetohydrostatic Grad-Shafranov (GS) reconstruction technique. Our analysis shows that there is a possibility of dipolarization fronts to originate from highly dissipated flux ropes which are in the late stage of their evolution, subjected to a continuous magnetic deterioration due to the reconnection process. These results may improve our understanding of magnetoplasma processes in Earth's magnetotail.
\end{abstract}

Keywords. Magnetospheric physics (magnetospheric configuration and dynamics; magnetotail; storms and substorms)

\section{Introduction}

Dipolarization fronts are a common feature of substorm dynamics routinely observed in the Earth's magnetotail. They are related to magnetic reconnection and are embedded into reconnection-driven sporadic bursty bulk flows (BBFs) (Angelopoulos et al., 1992, 1996; Nakamura et al., 2001). Earthward-traveling dipolarization fronts are a signature of the change of the magnetic field configuration from stretched to more dipolarized, demonstrating a sharp, large-amplitude increase in the $z$ component of the magnetic field. Dipolarization fronts are also known in the literature as plasma bubbles (Birn et al., 2011; Sergeev et al., 1996; Chen and Wolf, 1993; Pontius and Wolf, 1990; Walsh et al., 2009). Plasma bubbles are depleted flux tubes which have lower entropy than the adjacent flux tubes and are described by the adiabatic equation of state $P V^{\gamma} \cdot \gamma$ is the ratio of specific heats, $P$ is the plasma pressure within the flux tube, and $V=\int(1 / B) \mathrm{d} s$ is the volume of the flux tube containing $1 \mathrm{~Wb}$ of magnetic flux, where $s$ is the length of the flux tube. They reflect the properties of reconnection regions they originate from and thus are of key importance for our understanding of magnetospheric substorm dynamics, and this is the reason why there is a plethora of studies investigating these plasma structures both theoretically/numerically (Sitnov and Swisdak, 2011; Nakamura et al., 2002; Birn et al., 2011; Sitnov et al., 2009; Guzdar et al., 2010) and observationally (Nakamura et al., 2001, 2002; Runov et al., 2009; Sergeev et al., 2009; Slavin et al., 2003a, b), just to mention but a few.

Earthward- and tailward-traveling flux ropes, on the other hand, are also commonly observed in the magnetotail. They are characterized by helical magnetic configuration and are the consequence of multiple $\mathrm{X}$-line formations due to magnetotail reconnection process (Slavin et al., 2003a). Their most prominent in situ feature is a bipolar $B_{z}$ signature, coincident with a peak in the $B_{y}$ component which corresponds to their strong core field, and they are registered frequently in the near-tail region, from 15 to $30 R_{\mathrm{E}}$ (Earth radii) (Slavin et al., 2003a, b). In particular, earthward moving flux ropes have a well-defined $\mp B_{z}$ signature and are generally observed in regions where high-speed earthward plasma flows occur. 
A fundamental issue in space science and space weather is to understand the transport of solar wind plasma throughout the terrestrial magnetosphere and particularly to answer the question of how the energy from the solar wind entering into the terrestrial magnetospheric system finally gets dissipated via the magnetospheric substorm phenomenon (Koskinen, 2011). Therefore, the global concept of magnetospheric substorms has become a focus for much of the magnetospheric research that occurs today. This intense research interest and the need to shed light on the magnetotail process responsible for substorm onset and evolution eventually led to the realization of the THEMIS (Time History of Events and Macroscale Interactions during Substorms; Angelopoulos, 2008) mission, whose primary goal is to refine our knowledge regarding the substorm process and its onset triggering mechanism. As was stated by THEMIS principal investigator in Angelopoulos (2008), "substorms represent a fundamental mode of global magnetospheric circulation, a macroscopic instability whose phenomenological and theoretical understanding is crucial for space science, basic plasma physics and space weather" and as such many studies have tried to decipher the mechanism of substorm instability (Lui, 2001, 2003, 2004; Angelopoulos et al., 2008; Baker et al., 1996; Shiokawa et al., 1998).

In this case-based study, we report on a number of earthward dipolarization fronts and flux ropes identified during THEMIS tail seasons (Table 1) and analyze two representative events registered at $-9.8 R_{\mathrm{E}}$ (near-Earth location, where flow braking and diversion usually happens; e.g., Keika et al., 2009; Shiokawa et al., 1997; Yao et al., 2012) and $-15.7 R_{\mathrm{E}}$ (downtail location, where usually a near-Earth neutral line is formed; Cao et al., 2012, and references therein). For the selection of the events we did not use any automated selection algorithm. Instead, we went through all the THEMIS tail seasons and examined panel by panel the online THEMIS summary plots and particularly the magnetic field and plasma velocity components $B_{z}$ and $V_{x}$, respectively. By comparing the two events' observational features we propose a scenario of how earthward-traveling magnetic disturbances, commonly characterized as flux ropes, would be inevitably deteriorated due to reconnection process, thus falling from flux rope state to dipolarization front state. In the context of this study, we define deterioration as the continuous process of peeling off the outer layers of the flux rope due to magnetic field reconnection until full annihilation of the flux rope. The data analysis results and the phenomenological model presented to interpret the near-Earth event may place a new interpretation context concerning the nature of (a) the dipolarization fronts and (b) the triggering mechanism for substorm onset initiation.

\section{Analysis and results}

The THEMIS mission was launched on 17 February 2007 and consists of five identical spacecraft equipped with instruments that measure particles and fields (Angelopoulos, 2008). In this study we make use of $3 \mathrm{~s}$ time resolution plasma data obtained from the ESA (electrostatic analyzer; McFadden et al., 2008) instrument, while for the magnetic field data provided by the FGM (fluxgate magnetometer; Auster et al., 2008) instrument we make use of 0.25 and $3 \mathrm{~s}$ time resolutions.

The Grad-Shafranov (GS) (Hau and Sonnerup, 1999; Hu and Sonnerup, 2002; Sonnerup et al., 2006; Möstl et al., 2009; Isavnin et al., 2011; Teh et al., 2014) reconstruction method is applied to recover the two-dimensional magnetic field maps of the events. The technique assumes that the analyzed magnetic structure is in magnetohydrostatic equilibrium $(\nabla p=\boldsymbol{j} \times \boldsymbol{B})$, and is $21 / 2$-dimensional, i.e., the structure has translational symmetry along its invariant axis $\left(\frac{\partial}{\partial z}=0\right)$. The GS reconstruction is applied under force-free conditions $(\boldsymbol{j} \times \boldsymbol{B}=0)$. The choice of the force-free approach is partly imposed by the limitation of the maximum time resolution of the plasma data obtained from the ESA instrument. In a previous study by Slavin et al. (2003a), it was demonstrated that the magnetic field measurements of the flux ropes in the near tail can be successfully fitted with force-free flux rope models, thus suggesting that the observed flux ropes propagating towards the Earth are truly force free. This suggestion was further supported by the application of the curlometer technique to a flux rope registered by the Cluster spacecraft in the magnetotail (Slavin et al., 2003b). The analysis showed that the current in the observed flux rope is largely field aligned and although the flux ropes in the magnetotail are not strictly force free the usage of the force-free approximation proves to be reasonable. The GS reconstruction in contrast to most of the flux rope fitting models is more robust for the analysis of the flux ropes that are perturbed or distorted by the media they are propagating through, since it is capable of automatically choosing only the inner part of the flux rope which is the least perturbed (Isavnin et al., 2011). This property of GS reconstruction is important for the current study since we consider the flux ropes registered near the Earth to be highly deteriorated and thus heavily perturbed. By applying this technique we are able to identify the topology of a magnetic field structure "frozen in the earthward moving plasma" (Roberts, 2007) based on in situ measurements, thus providing an estimate of the local geometrical orientation of the structure and an approximate map of the magnetic field and plasma in a region around the spacecraft trajectory. The reconstruction is performed in the de Hoffmann-Teller (HT) frame, in which the electric field vanishes and thus the analyzed structure can be considered time stationary $\left(\frac{\partial}{\partial t}=0\right)$, i.e., it does not evolve during the passage of the spacecraft through it. The quality of the HT frame is assessed by the correlation coefficient (cc) between the com- 
Table 1. Eight selected THEMIS events conventionally classified either as flux rope (FR) or dipolarization front (DF) registered at various radial distances.

\begin{tabular}{|c|c|c|c|c|c|c|c|}
\hline Date & Event time & THEMIS spacecraft & Location in GSM $\left(R_{\mathrm{E}}\right)$ & Conv. class. & Invariant axis in GSM & Residual & cc HT \\
\hline $19 / 1 / 2008$ & $\sim 03: 37 \mathrm{UT}$ & P3 (D) & $(-9.8,-0.5,-3.7)$ & DF & $(-0.867,0.341,0.362)$ & 2.503 & 0.963 \\
\hline $16 / 3 / 2008$ & $\sim 07: 55 \mathrm{UT}$ & P4 (E) & $(-10.5,4.9,-1.6)$ & DF & $(-0.412,-0.022,0.911)$ & 0.727 & 0.634 \\
\hline $17 / 6 / 2011$ & $\sim 13: 39 \mathrm{UT}$ & $\mathrm{P} 4(\mathrm{E})$ & $(-8.6,1.7,3.7)$ & DF & $(0.448,0.850,0.279)$ & 6.700 & 0.955 \\
\hline 23/7/2012 & $\sim 11: 37 \mathrm{UT}$ & P5 (A) & $(-9.8,3.2,2.8)$ & DF & $(0.983,0.140,-0.121)$ & 1.188 & 0.981 \\
\hline $9 / 3 / 2008$ & $\sim 07: 52 \mathrm{UT}$ & P1 (B) & $(-17.1,4.4,-1.2)$ & FR & $(-0.929,-0.369,0.008)$ & 0.723 & 0.950 \\
\hline $5 / 2 / 2009$ & $\sim 11: 02 \mathrm{UT}$ & $\mathrm{P} 2(\mathrm{C})$ & $(-17.5,-6.1,-3.3)$ & FR & $(-0.745,0.632,-0.211)$ & 0.784 & 0.997 \\
\hline $21 / 2 / 2009$ & $\sim 11: 32 \mathrm{UT}$ & $\mathrm{P} 2(\mathrm{C})$ & $(-15.7,-3.6,-2.3)$ & FR & $(-0.709,0.704,0.042)$ & 0.307 & 0.976 \\
\hline $13 / 3 / 2009$ & $\sim 05: 54 \mathrm{UT}$ & $\mathrm{P} 2(\mathrm{C})$ & $(-16.0,1.4,-1.0)$ & FR & $(-0.888,-0.459,0.004)$ & 0.443 & 0.955 \\
\hline
\end{tabular}

ponents of $-\boldsymbol{V}^{(m)} \times \boldsymbol{B}^{(m)}$ and $-\boldsymbol{V}_{\mathrm{HT}}^{(m)} \times \boldsymbol{B}^{(m)}$, where $m$ is the ordinal number in the data series.

The estimation of the local orientation of a flux rope, i.e., the invariant axis direction, is based on the assumption that the spacecraft crosses the same helical equipotential lines of the flux rope twice, when moving inward and outward of the flux rope. According to this assumption, the in situ measurements of the magnetic field and plasma properties along the trajectory of the spacecraft should ideally result in two coinciding branches of the $P_{t}(A)$ curve, where $P_{t}=p+B_{z}^{2} / 2 \mu_{0}$ is the transverse pressure, i.e., the pressure measured along the invariant axis of the flux rope, and $A(x, y)$ is the vector potential projected onto the invariant axis direction so that $\boldsymbol{A}=A(x, y) \hat{z}$. The level of coincidence of the branches of the $P_{t}(A)$ curve is measured by the residual coefficient $R$, which is calculated as the normalized 2-norm of the residual vector. The residual vector is the point-by-point pairwise difference of the branches of the $P_{t}(A)$ curve. The optimal direction of the invariant axis is characterized by a minimal value in $R$. The residual coefficient also reflects the level of the flux rope distortion, that is, the higher residue between the branches of the $P_{t}(A)$ curve for an optimal direction of the invariant axis is a signature of the bigger difference in plasma and magnetic field properties between different parts of the flux rope. We are using the residual coefficient in order to assess the level of deterioration of a flux rope and demonstrate how the deterioration level and hence the distortion of a flux rope differs amongst the downtail and near-Earth events. The higher the residual coefficient the more distorted is the flux rope.

Using the GS technique we studied four near-Earth events and four downtail events. The results of the analysis are presented in Table 1. The value of the residual coefficient is typically larger for the events registered near the Earth than for those registered downtail. This fact supports our flux rope deterioration-based scenario during propagation towards the Earth.

For the purposes of our study we have selected two representative events from the eight we have analyzed (Table 1) and compare them in order to demonstrate their similarities. The first event occurred during a moderate geomagnetic sub- storm with an auroral electrojet (AE) index reaching values more than $300 \mathrm{nT}$ and is conventionally classified as flux rope (21 February 2009 downtail event), while the second event occurred during a small geomagnetic substorm where the AE index ranged between 150 and $200 \mathrm{nT}$ and is conventionally classified as dipolarization front (23 July 2012 nearEarth event). Not taking into account the event registered on 16 March 2008 at $\sim$ 07:55 UT with a low-quality HT frame, each selected event has, as far as the residual coefficient is concerned, the smallest value in its category, but, as can be noticed, the value for the downtail event is almost four times smaller than that for the near-Earth event. The comparison allows us to draw conclusions concerning the nature of dipolarization fronts and their relation to earthward moving flux ropes.

\subsection{Downtail flux rope event}

On 21 February 2009 the average THEMIS C satellite location, for the time interval 11:31-11:34 UT, was [-15.7, $-3.6,-2.3] R_{\mathrm{E}}$, in geocentric solar magnetospheric (GSM) coordinates with the $X$ axis pointing from the Earth towards the Sun, the $Y$ axis pointing towards dusk, and the $Z$ axis lying on the plane containing the dipole axis. Figure $1 \mathrm{a}-\mathrm{h}$ give an overview of the magnetic field components in GSM coordinates, the magnetic field intensity, the calculated $V_{x}, V_{y}$, $V_{z}$ convective plasma velocities (plasma velocities perpendicular to magnetic field) also in GSM coordinates, and ion density. The clear signatures shown are those of an earthward propagating flux rope which is depicted schematically in the lower part of Fig. 1. THEMIS C's relative trajectory is denoted by the dashed green arrow. The main observational feature here corresponds to the time interval 11:32:35-11:32:45 UT, indicated by the vertical blue bar. During this interval, the $B_{z}$ magnetic field component turns southward obtaining negative values signifying the satellite's entrance into the leading portion of the flux rope structure. This trajectory portion is denoted in the schematic illustration by the horizontal blue bar. $B_{x}$ and $B_{y}$ demonstrate opposite behavior ( $B_{x}$ is decreasing while $B_{y}$ is increasing) implying that we enter in a region corresponding to the flux rope's core, as shown in the illustration. The flux rope is embedded in an earth- 

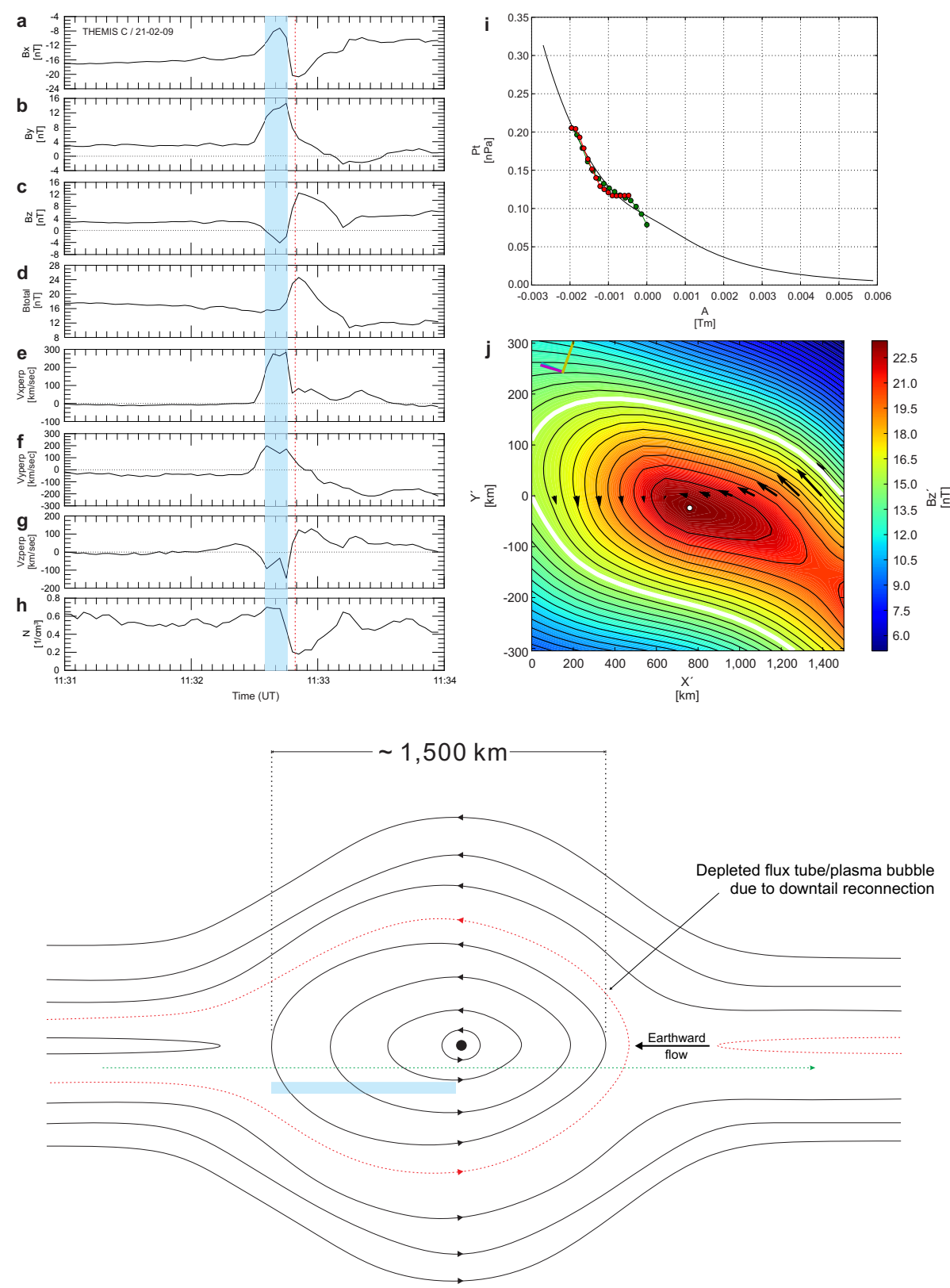

Figure 1. Downtail flux rope convected earthward observed by THEMIS C on 21 February 2009. Panels (a)-(h) show the three magnetic field components, magnetic field intensity, the three convective plasma velocity components, and plasma density. Panels (i) and (j) result from the GS reconstruction analysis. Panel (i) shows the two branches of the curve of the transverse magnetic pressure versus vector potential for the optimal direction of the invariant axis of the flux rope. The two branches correspond to the spacecraft movement inward and outward of the flux rope and the black curve is the analytical fit of $\operatorname{Pt}(A)$. Panel (j) shows the GS reconstructed magnetic field map of the flux rope cross section. $X^{\prime}, Y^{\prime}$ and $Z^{\prime}$ axes constitute the coordinate system of GS reconstruction, which is projected onto GSM coordinates as follows: $X^{\prime}=[-0.655,-0.680,0.329], Y^{\prime}=[0.260,0.205,0.943], Z^{\prime}=[-0.709,0.704,0.042]$. The white thick curve corresponds to the time interval used for GS reconstruction. The schematic illustration at the bottom of the figure is a 2-D cut in a plane containing the Earth and the apex of the flux rope, where the invariant axis is approximately perpendicular to this plane (and not necessarily where the spacecraft crossed the flux rope). In this regard, the earthward flow label denotes the velocity of the HT frame estimated in the apex of the flux rope.

ward convective plasma flow (panel e) which is also deflected duskward and southward indicative of a convective flow vortex (panels $f$ and $g$ ) due to the flux rope structure pileup with the upstream magnetic flux tubes. A particularly interesting observational feature is the gradual plasma density depletion (panel h) when $B_{z}$ reverses polarity implying the entrance into the flux rope's trailing portion. The dashed oval-shape red line in the schematic illustration denotes the depleted flux 
tube/plasma bubble that is traversed by the THEMIS satellite right at the time indicated by the dashed vertical red line shown in panels $\mathrm{a}-\mathrm{h}$, where its formation can be understood in terms of a downtail reconnection process. At this time instance, $B_{z}$ and $B_{\text {total }}$ obtain relatively high values with $B_{y}$ already decreased implying that we are exiting the flux rope with the magnetic field being dipolarized and compressed, thus leading to the asymmetric spatial configuration of the flux rope structure shown in the illustration.

By applying the Grad-Shafranov reconstruction technique (panels $i$ and $j$ ) for the time interval 11:32:42-11:32:47 UT, we verify that the observed magnetic field behavior can be explained in terms of a flux rope structure. Panel i shows the dependence of transverse magnetic pressure $P_{t}$ on vector potential when projected onto the plane perpendicular to the flux rope invariant axis direction. The two branches of the curve (red and green dots) correspond to the parts of the spacecraft trajectory inward and outward of the flux rope. Its spatial extent is estimated to be around $1500 \mathrm{~km}$, while its invariant axis lies predominantly on the $X Y-\mathrm{GSM}$ plane (see Table 1 and below). For this event, the axes orientations of the Grad-Shafranov frame, in GSM coordinates, were estimated to be $X^{\prime}=[-0.655,-0.680,0.329]$, $Y^{\prime}=[0.260,0.205,0.943], Z^{\prime}=[-0.709,0.704,0.042]$.

\subsection{Near-Earth flux rope event}

The same panels as in Fig. 1 are presented for THEMIS A in Fig. 2 during the period 11:35-11:40 UT on 23 July 2012 for an event that is characterized as a dipolarization front. In addition, energetic ion differential energy fluxes versus azimuthal angle in the probe spin plane, as measured by the solid-state telescope (SST) instrument in the $30-300 \mathrm{keV}$ energy range, are presented in panel i. During this time interval, the satellite was located near the Earth with an average location of $[-9.8,3.2,2.8] R_{\mathrm{E}}$, in GSM coordinates. The event demonstrates similar characteristics to those of the flux rope event analyzed previously. $B_{z}$ shows a clear polarity reversal from northward to southward and then again to northward (vertical blue bar). An interesting observational feature is the magnetic field intensity minimization reaching values around $6 \mathrm{nT}$, implying magnetic energy consumption and immediately afterwards its abrupt ejection coincident with an abrupt increase in $B_{z}$ indicating that the magnetic field is dipolarized and compressed, similar to downtail flux rope observations. As it is shown in panel i, several seconds before and during $B_{z}$ negative reversal, energetic ions superimposed over the preexisting plasma sheet population gradually appear streaming earthward and dawnward at $\sim-60^{\circ}$. This flow pattern is gradually becoming more earthward while approaching the magnetic structure ( $\pm B_{z}$ reversal). The structure is embedded in a high-speed earthward convective plasma flow with flow diversion present (panels $f$ and $g$ ) due to the structure's pileup with the upstream geomagnetic field (Keika et al., 2009).
The Grad-Shafranov reconstruction analysis (panels $j$ and k) for the time interval 11:37:26-11:37:33 UT provides a reasonable explanation that the structure under investigation, commonly classified as a dipolarization front, could originate from a magnetically deteriorated flux rope. Its invariant axis lies predominantly on the $X-\mathrm{GSM}$ axis (see Table 1 and below), while its limited spatial extent compared to the downtail flux rope, estimated to be around $800 \mathrm{~km}$, implies that it is continuously dissipated due to some kind of mechanism responsible for constantly eroding its outer magnetic shell. For this event, the axes orientations of the Grad-Shafranov frame, in GSM coordinates, were estimated to be $X^{\prime}=[-0.044,-0.458,-0.888]$, $Y^{\prime}=[-0.180,0.878,-0.444], Z^{\prime}=[0.983,0.140,-0.121]$. Regarding the invariant axis orientation, one could think that it should be in $Y$-GSM direction; however, the invariant axis need not be along the $Y$ direction. As a matter of fact, in a recent study Liu et al. (2013) pointed out that because the two edges of a flux rope are basically anchored to the ionosphere, plasma convection will drag such a structure in a way that will transform it eventually into a slingshot-shaped structure where the axial direction of the curved part of the flux rope may be parallel to $X-\mathrm{GSM}$ direction.

Our phenomenological model along with its key features is shown in the lower part of Fig. 2. The vertical red dashed line signifies the time when the THEMIS satellite enters into the depleted flux tubes/plasma bubbles formed upstream by the reconnection process. The dashed green arrow denotes the relative trajectory of THEMIS A through the flux rope.

\section{Discussion and conclusions}

A first attempt to decipher the process behind near-Earth flux rope dissipation was made by Vogiatzis et al. (2011), where reconnection was proposed as the responsible mechanism for flux rope deterioration. In that study it was proposed that the continuous process of peeling off the outer layers of the flux ropes due to magnetic field reconnection with the background geomagnetic field leads to magnetic field annihilation and magnetic energy consumption, which in turns powers a dawnward electric field responsible for ejecting ions in the dawnward direction.

The presented observations suggest that the near-Earth magnetic disturbance propagating earthward can be explained in terms of a flux rope structure embedded in fast plasma-sheet flow following the onset of BBF. The fact that the positive $B_{z}$ enhancement shows much larger value can be understood in terms of magnetic field compression being much more pronounced at the trailing edge of the flux rope due to the high-speed earthward convective flow, which pushes the flux rope against the geomagnetic field. The whole essence is the following: if we do observe earthward moving flux ropes at $\sim 15-18 R_{\mathrm{E}}$, why do we tend to avoid their existence at smaller radial distances? Is it because flux ropes 

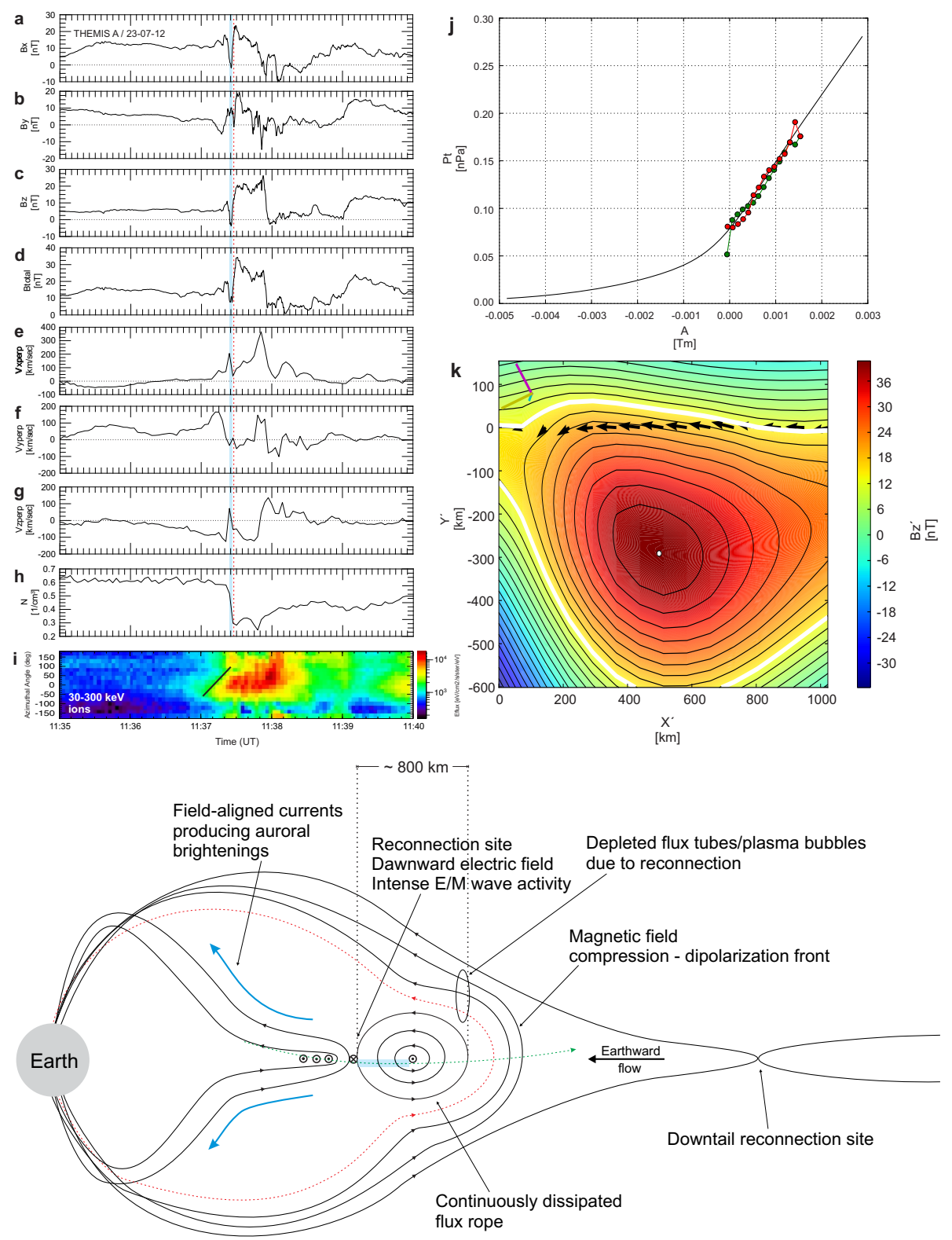

Figure 2. Near-Earth flux rope convected earthward observed by THEMIS A on 23 July 2012. Same format as in Fig. 1. The GS reconstruction frame is projected onto GSM coordinates as follows: $X^{\prime}=[-0.044,-0.458,-0.888], Y^{\prime}=[-0.180,0.878,-0.444], Z^{\prime}=$ $[0.983,0.140,-0.121]$. Panel (i) presents ion differential energy fluxes versus azimuthal angle in the probe rotation plane, measured by the solid-state telescope (SST) instrument in the 30-300 keV energy range. Zero degrees correspond to earthward fluxes. The schematic illustration at the bottom of the figure is a qualitative representation of the substorm model we propose. The illustration is formed by a 2-D cut in a plane containing the Earth and the apex of the flux rope, where the invariant axis is approximately perpendicular to this plane (and not necessarily where the spacecraft crossed the flux rope). In this regard, the earthward flow label denotes the velocity of the HT frame estimated in the apex of the flux rope.

tend to penetrate and finally get dissipated in the inner magnetosphere, thus obtaining a different form than the usual; the form of dipolarization fronts? We support the idea that flux ropes get dissipated on their way to the Earth and eventually converted to compression regions where magnetic field intensity is significantly elevated. Whether we observe a bipolar $B_{z}$ or not depends on the degree of flux rope dissipa- tion and where it was initially formed. Hence, dipolarization fronts can be observed at a wide range of radial distances in the Earth's magnetotail depending on the dissipation degree of the associated flux ropes. Moreover, earthward convected flux ropes can be initially formed far from or very close to the Earth, depending on where multiple X-lines are formed. This fact may have important implications for 
substorm-associated particle injections routinely observed by geostationary satellites and also for storm-time ring current buildup, by activating an efficient particle acceleration mechanism related to induced impulsive electric fields (Daglis and Kamide, 2004) whose location moves progressively earthward.

The ion injection earthward and dawnward shown in Fig. 2 (near-Earth THEMIS A event, panel i) could be understood in terms of this particle acceleration mechanism. According to this mechanism, ions being originally demagnetized, decouple from the magnetic field following their inertia and get accelerated by any electric field that would be present, e.g., the dawnward electric field. After executing meandering orbits across the current sheet (Speiser-type trajectories) (Speiser, 1965; Büchner and Zelenyi, 1989; Speiser, 1991), in the presence of a small southward magnetic field combined with the dawnward electric field they would be eventually ejected from it towards the Earth. As the flux ropes are approaching THEMIS A, the earthward-dawnward flow patterns start to diminish into more earthward ones implying that the energetic ions are observed right at the time of their generation meaning that they have not been injected upstream of the flux ropes yet (black oblique line in Fig. 2i). A synopsis of the above interpretation is shown in Fig. 3.

The possibility that BBF-type flux ropes carried towards the Earth and pushed up against the geomagnetic field will probably get dissipated due to reconnection with the geomagnetic field was first brought to attention by Slavin et al. (2003a). This study addressed the fundamental question regarding the fate of the earthward moving flux ropes in the Earth's plasma sheet. They surmised that the process of reconnection reduces the amount of southward magnetic flux along the earthward side of the flux ropes, a hypothesis that turns out to be fairly reasonable based on the deterioration mechanism shown in the present study.

Although in the present study we show the possibility of dipolarization fronts to originate from earthward moving, highly dissipated flux ropes, other explanations on the nature of these structures were previously presented. Southnorth rotation of $B_{z}$ magnetic field component is often interpreted as a transient profile of a remote $\mathrm{X}$-line due to its temporal change in the reconnection rate (Sergeev et al., 2005; Sitnov et al., 2009). Sormakov and Sergeev (2008) suggested that compression of the background magnetic field by a reconnected magnetic flux tube moving earthward can cause the bipolar variation of $B_{z}$. The strong core field $B_{y}$ of these structures can be explained by three-dimensional (3D) guide-field reconnection as demonstrated in magnetohydrodynamic (MHD) simulations by Shirataka et al. (2006). Later, Hasegawa et al. (2007) used the results of these MHD simulations for GS reconstruction analysis and showed that this technique can reconstruct flux ropes in the configurations without flux ropes being actually present. The latter means that one should be cautious when interpreting GS reconstruction results. Hasegawa et al. (2007) summed up a guideline that can be used to distinguish flux ropes from a 3$\mathrm{D}$ guide-field reconnection configuration. According to that guideline, the feature not typical for flux ropes is the presence of perpendicular velocity components in the HT frame, which is an indication of temporal variation of the structure, and plasma pressure enhancement in the leading part of the structure. Among the events in our list only the one registered on 16 March 2008 at $\sim$ 07:55 UT was characterized by low quality of HT with $\mathrm{cc}=0.634$, and hence could be a case of 3 -D guide-field reconnection. For all other events the value of cc is close to unity, which means that the temporal variations of those structures were low. While 3-D guide-field reconnection is reproducible in MHD simulations, it is a very special type of magnetic field configuration which cannot explain the majority of dipolarization fronts. For instance, this configuration requires the core field enhancement to be of the same sign as the cross-tail guide field $B_{y}$. However, there is no correlation found between the core field of the earthward moving flux-rope-like structures and the direction of the background $B_{y}$ field (Borg et al., 2012).

The phenomenological model introduced in this study provides a simple explanation of the so-called "pressure balance inconsistency" or "pressure crisis" problem first noted by Erickson and Wolf (1980), since it does not involve the arrival of flux tubes from the distant tail to the near-Earth region. To the contrary, in our model low entropy bubbles/dipolarization fronts are formed from the reconnection of the stretched geomagnetic field with the earthward moving flux ropes leading to the generation of reconnected flux tubes of increased volume $V$ and low plasma density. The pressure balance inconsistency stems from the fact that the ratio $\beta$ of particle pressure to magnetic pressure obtains unduly high values in the near-Earth equatorial plane when a flux tube shortens considerably as it convects earthward from -60 to $-10 R_{\mathrm{E}}$ (Erickson and Wolf, 1980), assuming adiabatic compression of plasma during the earthward magnetoplasma transport.

A number of phenomenological models have been developed to describe the magnetospheric phenomena that compose a magnetospheric substorm. The two most prevailing substorm models, which suggest different standpoints on the substorm onset cause and location in the magnetotail, are the near-Earth neutral line (NENL) model (McPherron et al., 1973; Russell and McPherron, 1973; Baker et al., 1996) and the tail current disruption (TCD) model (Lui, 1991, 1996). The NENL model relies on dayside merging and magnetotail reconnection to explain the substorm cycle. The unique feature of this model is the formation of an X-line in the central plasma sheet at which plasma sheet closed field lines reconnect. Main observational evidence of this model are the nearEarth neutral line formation, BBFs transporting flux earthward, and plasmoid release downtail. This model places the substorm onset at the location of the near-Earth neutral line, at a downtail distance of $20-30 R_{\mathrm{E}}$.

Complementary to the NENL model, the flux pileup model (Shiokawa et al., 1998; Baumjohann et al., 1999) describes 


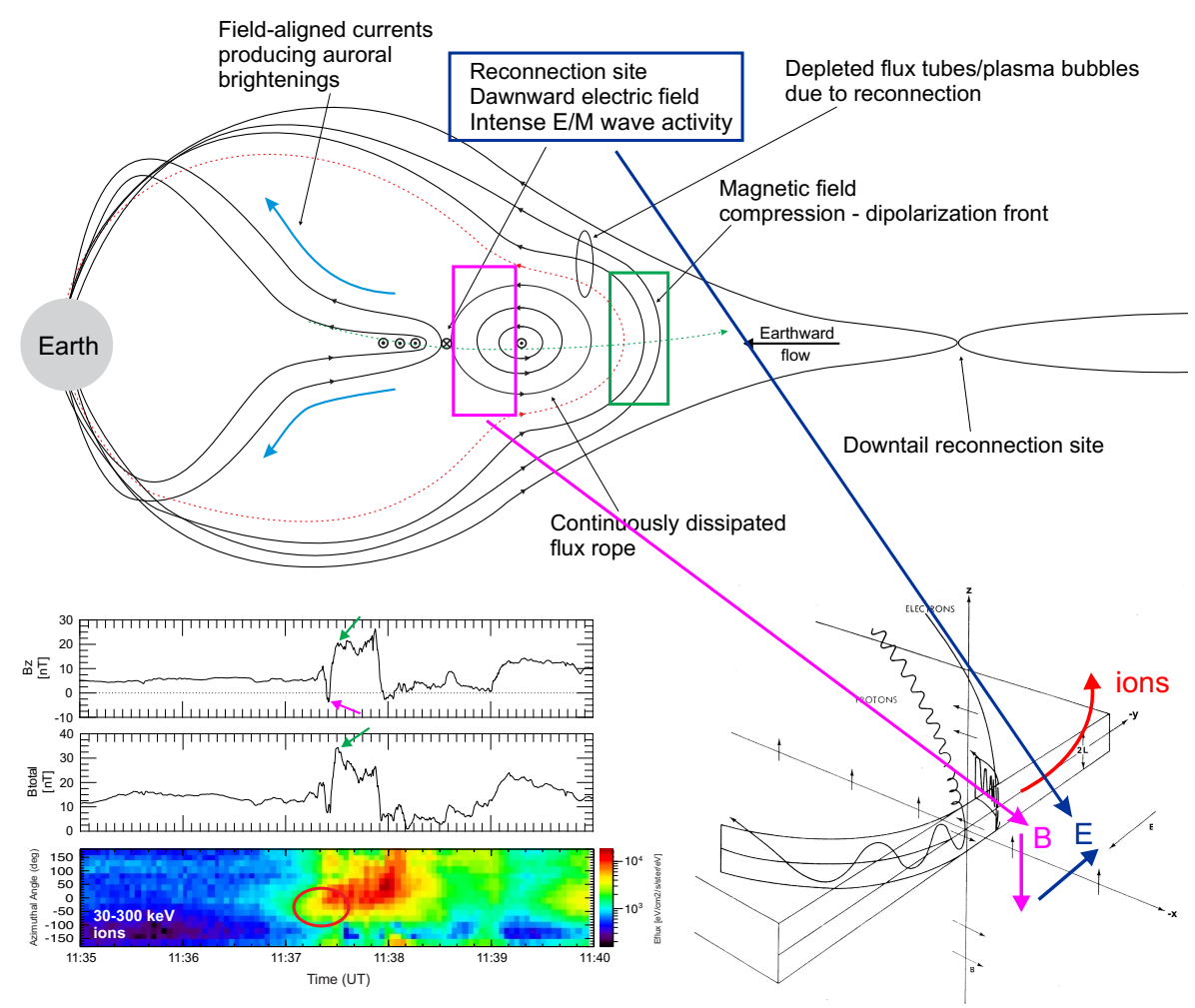

Figure 3. Synopsis of our interpretation in the context of the proposed model. Red oval denotes the earthward and dawnward injected energetic ions under the combined effect of a southward magnetic field and dawnward electric field. Key features of the interpretation are color coded. The bottom-right part of the figure is taken from Speiser (1965).

how magnetic flux is convected earthward from the neutral line, introducing the notion of a dawnward inertia current due to plasma flow deceleration as magnetic flux is piled up in the region where the magnetic configuration changes from tail-like to dipolar. As dipolarization fronts embedded in fast earthward BBFs generated by near-Earth reconnection soon encounter the strong dipolar field of the inner magnetosphere, an enhancement/pileup of the northward magnetic flux of the background dipole magnetic field is taking place. The ensuing tailward pressure force at the transition region between dipolar and tail-like magnetic field configurations has the effect of decelerating the earthward flows. This flow braking is accompanied by the generation of a dawnward cross-tail current at the stopping point. Observations support this hypothesis, as fast flows are seen less frequently closer to Earth. As the pileup proceeds, the region of dipolarization extends tailward. The tailward motion of the braking point thus corresponds to the poleward expansion of the aurora.

As has been shown previously by Vogiatzis et al. (2005), while analyzing a well-isolated substorm event, the stopping point at which earthward plasma flow is expected to be fully stopped was placed at a near-Earth distance of $4.3 R_{\mathrm{E}}$, well inside the geosynchronous orbit. This breaking point is expected to coincide with the initial stage of substorm expansion phase (Shiokawa et al., 1998). Furthermore, based on observational evidence from GOES and POLAR satellites, in the same study, it was clearly shown that substorm onset/dipolarization signatures are propagating tailward. In this regard, the plasma flow stopping point is expected to be the closest earthward point that earthward moving plasmoids can penetrate until they are fully dissipated and this signals the initial auroral brightening related to the initial stage of substorm expansion phase/substorm onset. After that time, the continuous supply with flux ropes and the accumulation of the resulting dipolarization fronts is what causes the progressive movement of field dipolarization tailward.

On the other hand, the tail current disruption model places the onset location near the Earth, at roughly $10 R_{\mathrm{E}}$, due to some instability reducing the cross-tail current. A suggested instability is the kinetic cross-field-streaming instability, producing lower hybrid waves Lui et al. (1990). As the plasma sheet thins near its earthward edge during the substorm growth phase, the cross-tail current sheet thickness decreases to a length scale comparable to the thermal ion gyroradius, and the neutral sheet ions become unmagnetized and begin to stream across the current sheet in serpentine (Speiser) orbits. Electrons have smaller gyroradius and are more closely tied to the magnetic field lines, and hence they remain magnetized. The relative drift between electrons and 
ions provides energy to excite lower hybrid waves which in turn trigger tail current disruption.

The tail current disruption model suggests a diversion of the cross-tail current, that is, formation of the substorm current wedge (SCW), as the cross-tail current is prevented from flowing across the tail. According to a number of observations (Lopez et al., 1988, b; Lopez and Lui, 1990), the current sheet disruption begins close to geosynchronous orbit and expands radially outward into the tail. Such observations have led to the conclusion that the substorm expansion does not involve reconnection on the open field lines of the tail lobe. It has been suggested, instead, that the tail current disruption launches a rarefaction wave which moves tailward and produces multiple sites of tail current disruption. The passing of rarefaction wave leaves a thin current sheet with reduced magnetic field normal to the neutral sheet. At some point down the tail, late in the expansion phase, or perhaps at the beginning of the recovery phase, these effects initiate reconnection and the subsequent generation and tailward release of a plasmoid.

In the substorm model we propose (referred to in the following as the CDXL model, which stands for current disruption $\mathrm{X}$-line model) collisionless reconnection, where flux tubes merge and magnetic flux of opposite field direction annihilates, has a dual role since it is responsible both for flux rope energy dissipation/tail current disruption and multiple $\mathrm{X}$-lines and NENL formation. In the CDXL model, nearEarth tail current disruption and X-lines/NENL formation act synergistically, that is, the latter producing the earthwardtraveling flux ropes and the associated BBFs and the former reconfiguring the magnetic field topology from tail-like to dipole-like via magnetic flux rope energy dissipation due to an ongoing merging of the vertically oriented, antiparallel magnetic field lines. Thus, driven reconnection at the earthward edge of the magnetic flux ropes with the background geomagnetic field is a purely classical process, where released power in a merging event is proportional to the magnetic field strength and the length of flux tubes contact $\ell_{\|}$ along the field lines. Moreover, the annihilation process and the released power are sensitive functions of the angle under which the field lines come into contact (Treumann et al., 2012). This process inevitably leads to the continuous disruption of the cross-tail current and the generation of SCW field aligned currents (FACs).

Under the CDXL model the dawnward inertia current can be thought of as the dawnward current sheet generated by the merging of the oppositely directed, vertically oriented field lines of the near-Earth stretched magnetotail and the flux ropes front side at the reconnection site, with the outer layers of the flux ropes being essentially stripped away as they travel further earthward. The geomagnetic field erosion and its subsequent reconfiguration into a dipolar geometry through flux rope merging is what leads to cross-tail current disruption and current wedge formation, not the reverse process. Furthermore, the dipolarization fronts being observed are nothing else but compressed dipolarized flux tubes originating from the merging of the outer layers of the flux ropes, that is, the compressed trailing edges of the deteriorated flux ropes embedded inside the BBFs.

The CDXL model providing a unified context where NENL and TCD models are combined in a consistent manner is presented schematically in the drawing of Fig. 2, where the main phenomena taking place during a substorm event are shown. The drawing in Fig. 2 depicts only an instant in time. Whether the magnetic field remains dipolarized or not depends heavily on two competing processes: (1) that of the magnetic flux convection adding magnetic energy to the lobes of the magnetotail due to dayside merging, creating an increased current in the neutral sheet and thinning/stretching of the magnetotail and (2) that of multiple flux rope formation and merging with the geomagnetic field disrupting the cross-tail current and thus dipolarizing the magnetic field. During the recovery phase of a substorm where flux convection starts to cease, the second process starts to prevail leading to the final dipolar reconfiguration of the magnetosphere.

The CDXL model places the substorm onset at a nearEarth distance of 5-8 $R_{\mathrm{E}}$, which progressively moves tailward (Vogiatzis et al., 2005) as earthward moving flux ropes get absorbed by the geomagnetic field through reconnection process. This mechanism is consistent with the motion of auroral enhancement (known as auroral breakup) traveling northward and westward (Akasofu, 1964; Baker et al., 2002; Henderson et al., 2006). Moreover, whether there is only a sequence of auroral pseudobreakups or a full substorm expansion, manifested by the dramatic brightening of auroral features, directly depends on the occurrence frequency of flux ropes moving earthward and consequently their ability of temporarily or permanently disrupting the cross-tail current.

Acknowledgements. I. I. Vogiatzis sincerely thanks the THEMIS Principal Investigator V. Angelopoulos and the THEMIS team for their open data and processing software policy. This research has been co-financed by the National Natural Science Foundation of China (NSFC), grant 41150110579, the European Union (European Social Fund - ESF) and Greek national funds through the Operational Program "Education and Lifelong Learning" of the National Strategic Reference Framework (NSRF) - Research Funding Program: Thales. Investing in knowledge society through the European Social Fund. The work of A. Isavnin was supported by the Academy of Finland.

Topical Editor E. Roussos thanks three anonymous referees for their help in evaluating this paper. 


\section{References}

Akasofu, S.-I.: The development of the auroral substorm, Planet. Space Sci., 12, 273-282, 1964.

Angelopoulos, V.: The THEMIS mission, Space Sci. Rev., 141, 534, 2008.

Angelopoulos, V., Baumjohann, W., Kennel, C. F., Coroniti, F. V., Kivelson, M. G., Pellat, R., Walker, R. J., Lühr, H., and Paschmann, G.: Bursty bulk flows in the inner central plasma sheet, J. Geophys. Res., 97, 4027-4039, 1992.

Angelopoulos, V., Coroniti, F. V., Kennel, C. F., Kivelson, M. G., Walker, R. J., Russell, C. T., McPherron, R. L., Sanchez, E., Meng, C.-I., Baumjohann, W., Reeves, G. D., Belian, R. D., Sato, N., Friis-Christensen, E., Sutcliffe, P. R., Yumoto, K., and Harris, T.: Multipoint analysis of a bursty bulk flow event on April 11, 1985, J. Geophys. Res., 101, 4967-4989, 1996.

Angelopoulos, V., McFadden, J. P., Larson, D., Carlson, C. W., Mende, S. B., Frey, H., Phan, T., Sibeck, D. G., Glassmeier, K.H., Auster, U., Donovan, E., Mann, I. R., Rae, I. J., Russell, C. T., Runov, A., Zhou, X.-Z., and Kepko, L.: Tail reconnection triggering substorm onset, Science, 321, 931-935, 2008.

Auster, H. U., Glassmeier, K. H., Magnes, W., Aydogar, O., Baumjohann, W., Constantinescu, D., Fischer, D., Fornacon, K. H., Georgescu, E., Harvey, P., Hillenmaier, O., Kroth, R., Ludlam, M., Narita, Y., Nakamura, R., Okrafka, K., Plaschke, F., Richter, I., Schwarzl, H., Stoll, B., Valavanoglou, A., and Wiedemann, M.: The THEMIS Fluxgate Magnetometer, Space Sci. Rev., 141, 235-264, 2008.

Baker, D. N., Pulkkinen, T. I., Angelopoulos, V., Baumjohann, W., and McPherron, R. L.: Neutral line model of substorms: Past results and present view, J. Geophys. Res., 101, 12975-13010, 1996.

Baker,D. N., Peterson, W. K., Eriksson, S., Li, X., Blake, J. B., Burch, J. L., Daly, P. W., Dunlop, M. W., Korth, A., Donovan, E., Friedel, R., Fritz, T. A., Frey, H. U., Mende, S. B., Roeder, J., and Singer, H. J.: Timing of magnetic reconnection initiation during a global magnetospheric substorm onset, Geophys. Res. Lett., 29, 2190-2193, 2002.

Baumjohann, W., Hesse, M., Kokubun, S., Mukai, T., Nagai, T., and Petrukovich, A. A.: Substorm dipolarization and recovery, J. Geophys. Res., 104, 24995-25000, 1999.

Birn, J., Nakamura, R., Panov, E. V., and Hesse, M.: Bursty bulk flows and dipolarization in MHD simulations of magnetotail reconnection, J. Geophys. Res., 116, A01210, doi:10.1029/2010JA016083, 2011.

Borg, A. L., Taylor, M. G. G. T., and Eastwood, J. P.: Observations of magnetic flux ropes during magnetic reconnection in the Earth's magnetotail, Ann. Geophys., 30, 761-773, doi:10.5194/angeo-30-761-2012, 2012.

Büchner, J. and Zelenyi, L.: Regular and chaotic charged particle motion in magnetotaillike field reversals: 1 . Basic theory of trapped motion, J. Geophys. Res., 94, 11821-11842, 1989.

Cao, X., Pu, Z. Y., Du, A. M., Mishin, V. M., Wang, X. G., Xiao, C. J., Zhang, T. L., Angelopoulos, V., McFadden, J. P., and Glassmeier, K. H.: On the retreat of near-Earth neutral line during substorm expansion phase: a THEMIS case study during the 9 January 2008 substorm, Ann. Geophys., 30, 143-151, doi:10.5194/angeo-30-143-2012, 2012.

Chen, C. X. and Wolf, R. A.: Interpretation of high-speed flows in the plasma sheet, J. Geophys. Res., 98, 21409-21419, 1993.
Daglis, I. A. and Kamide, Y.: The Role of Substorms in Storm-time Particle Acceleration, in: Disturbances in Geospace: The StormSubstorm Relationship, edited by: Sharma, A. S., Kamide, Y., and Lakhina, G. S., Vol. 142 of Geophys. Monogr. Ser., 119-129, AGU, 2004.

Erickson, G. M. and Wolf, R. A.: Is steady convection possible in the Earth's magnetotail?, Geophys. Res. Lett., 7, 897-900, 1980.

Guzdar, P. N., Hassam, A. B., Swisdak, M., and Sitnov, M. I.: A simple MHD model for the formation of multiple dipolarization fronts, Geophys. Res. Lett., 37, L20102, doi:10.1029/2010GL045017, 2010.

Hasegawa, H., Nakamura, R., Fujimoto, M., Sergeev, V. A., Lucek, E. A., Rème, H., and Khotyaintsev, Y.: Reconstruction of a bipolar magnetic signature in an earthward jet in the tail: Flux rope or 3D guide-field reconnection?, J. Geophys. Res., 112, A11206, doi:10.1029/2007JA012492, 2007.

Hau, L.-N. and Sonnerup, B. U. Ö.: Two-dimensional coherent structures in the magnetopause: Recovery of static equilibria from single-spacecraft data, J. Geophys. Res., 104, 6899-6917, 1999.

Henderson, M. G., Reeves, G. D., Skoug, R., Thomsen, M. F., Denton, M. H., Mende, S. B., Immel, T. J., Brandt, P. C., and Singer, H. J.: Magnetospheric and auroral activity during the 18 April 2002 sawtooth event, J. Geophys. Res., 111, A01S90, doi:10.1029/2005JA011111, 2006.

$\mathrm{Hu}, \mathrm{Q}$. and Sonnerup, B. U. Ö.: Reconstruction of magnetic clouds in the solar wind: Orientations and configurations, J. Geophys. Res., 107, SSH10.1-SSH10.15, doi:10.1029/2001JA000293, 2002.

Isavnin, A., Kilpua, E. K. J., and Koskinen, H. E. J.: Grad-Shafranov Reconstruction of Magnetic Clouds: Overview and Improvements, Sol. Phys., 273, 205-219, 2011.

Keika, K., Nakamura, R., Volwerk, M., Angelopoulos, V., Baumjohann, W., Retinò, A., Fujimoto, M., Bonnell, J. W., Singer, H. J., Auster, H. U., McFadden, J. P., Larson, D., and Mann, I.: Observations of plasma vortices in the vicinity of flow-braking: a case study, Ann. Geophys., 27, 3009-3017, doi:10.5194/angeo27-3009-2009, 2009.

Koskinen, H. E. J.: Physics of space storms - From the solar surface to the Earth, Springer - Praxis, Berlin Heidelberg, 2011.

Liu, C., Feng, X., Guo, J., and Ye, Y.: Study of small-scale plasmoid structures in the magnetotail using Cluster observations and Hall MHD simulations, J. Geophys. Res., 118, 2087-2100, 2013.

Lopez, R. E. and Lui, A. T. Y.: A multisatellite case study of the expansion of a substorm current wedge in the near-Earth magnetotail, J. Geophys. Res., 95, 8009-8017, 1990.

Lopez, R. E., Sibeck, D. G., Lui, A. T. Y., Takahashi, K., and McEntire, R. W.: Substorm variations in the magnitude of the magnetic field: AMPTE/CCE observations, J. Geophys. Res., 93, 1444414452, 1988.

Lopez, R. E., Baker, D. N., Lui, A. T. Y., Sibeck, D. G., and Belian, R. D.: The radial and longitudinal propagation characteristics of substorm injections, Adv. Space Res., 8, 91-95, 1988 b.

Lui, A. T. Y.: A synthesis of magnetospheric substorm models, J. Geophys. Res., 96, 1849-1856, 1991.

Lui, A. T. Y.: Current disruption in the Earth's magnetosphere: Observations and models, J. Geophys. Res., 101, 13067-13088, 1996. 
Lui, A. T. Y.: A multiscale model for substorms, Space Sci. Rev., 95, 325-345, 2001.

Lui, A. T. Y.: Cause of magnetospheric substorms, Plasma Phys. Control. F., 45, 841-852, 2003.

Lui, A. T. Y.: Potential plasma instabilities for substorm expansion onsets, Space Sci. Rev., 113, 127-206, 2004.

Lui, A. T. Y., Mankofsky, A., Chang, C.-L., Papadopoulos, K., and Wu, C. S.: A current disruption mechanism in the neutral sheet: A possible trigger for substorm expansions, Geophys. Res. Lett., 17, 745-748, 1990.

McFadden, J. P., Carlson, C. W., Larson, D., Bonnell, J., Mozer, F., Angelopoulos, V., Glassmeier, K.-H., and Auster, U.: THEMIS ESA First Science Results and Performance Issues, Space Sci. Rev., 141, 477-508, 2008.

McPherron, R. L., Russell, C. T., and Aubry, M. P.: Satellite studies of magnetospheric substorms on August 15, 1968; 9. Phenomenological model for substorms, J. Geophys. Res., 78, 31313149, 1973.

Möstl, C., Farrugia, C. J., Biernat, H. K., Leitner, M., Kilpua, E. K. J., Galvin, A. B., and Luhmann, J. G.: Optimized Grad - Shafranov Reconstruction of a Magnetic Cloud Using STEREO-Wind Observations, Sol. Phys., 256, 427-441, 2009.

Nakamura, R., Baumjohann, W., Schödel, R., Brittnacher, M., Sergeev, V. A., Kubyshkina, M., Mukai, T., and Liou, K.: Earthward flow bursts, auroral streamers, and small expansions, J. Geophys. Res., 106, 10791-10802, 2001.

Nakamura, R., Baumjohann, W., Klecker, B., Bogdanova, Y., Balogh, A., Rème, H., Bosqued, J. M., Dandouras, I., Sauvaud, J. A., Glassmeier, K.-H., Kistler, L., Mouikis, C., Zhang, T. L., Eichelberger, H., and Runov, A.: Motion of the dipolarization front during a flow burst event observed by Cluster, Geophys. Res. Lett., 29, 1942, doi:10.1029/2002GL015763, 2002.

Pontius, D. H. J. and Wolf, R. A.: Transient flux tubes in the terrestrial magnetosphere, Geophys. Res. Lett., 17, 49-51, 1990.

Roberts, P. H.: Alfvén's Theorem and the Frozen Flux Approximation, Encyclopedia of Geomagnetism and Paleomagnetism, 711, 2007.

Runov, A., Angelopoulos, V., Sitnov, M. I., Sergeev, V. A., Bonnell, J., McFadden, J. P., Larson, D., Glassmeier, K.H., and Auster, U.: THEMIS observations of an earthwardpropagating dipolarization front, Geophys. Res. Lett., 36, L14106, doi:10.1029/2009GL038980, 2009.

Russell, C. T. and McPherron, R. L.: The magnetotail and substorms, Space Sci. Rev., 15, 205-266, 1973.

Sergeev, V. A., Angelopoulos, V., Gosling, J. T., Catell, C. A., and Russell, C. T.: Detection of localized, plasma-depleted flux tubes or bubbles in the midtail plasma sheet, J. Geophys. Res., 101, 10817-10826, 1996.

Sergeev, V. A., Kubyshkina, M. V., Baumjohann, W., Nakamura, R., Amm, O., Pulkkinen, T., Angelopoulos, V., Mende, S. B., Klecker, B., Nagai, T., Sauvaud, J.-A., Slavin, J. A., and Thomsen, M. F.: Transition from substorm growth to substorm expansion phase as observed with a radial configuration of ISTP and Cluster spacecraft, Ann. Geophys., 23, 2183-2198, doi:10.5194/angeo-23-2183-2005, 2005.

Sergeev, V., Angelopoulos, V., Apatenkov, S., Bonnell, J., Ergun, R., Nakamura, R., McFadden, J., Larson, D., and Runov, A.: Kinetic structure of the sharp injection/dipolarization front in the flow-braking region, Geophys. Res. Lett., 36, L21105, doi:10.1029/2009GL040658, 2009.

Shiokawa, K., Baumjohann, W., and Haerendel, G.: Breaking of high-speed flows in the near-Earth tail, Geophys. Res. Lett., 24, 1179-1182, 1997.

Shiokawa, K., Baumjohann, W., Haerendel, G., Paschmann, G., Fennell, J. F., Friis-Christensen, E., Lühr, H., Reeves, G. D., Russell, C. T., Sutcliffe, P. R., and Takahashi, K.: High-speed ion flow, substorm current wedge, and multiple Pi 2 pulsations, J. Geophys. Res., 103, 4491-4507, 1998.

Shirataka, N., Fujimoto, M., Hasegawa, H., and TanDokoro, R.: Reproducing the bipolar magnetic signature at the jet leading edge by three-dimensional reconnection with nonzero guide field, J. Geophys. Res., 111, A07201, doi:10.1029/2005JA011521, 2006.

Sitnov, M. I. and Swisdak, M.: Onset of collisionless magnetic reconnection in two-dimensional current sheets and formation of dipolarization fronts, J. Geophys. Res., 116, A12216, doi:10.1029/2011JA016920, 2011.

Sitnov, M. I., Swisdak, M., and Divin, A. V.: Dipolarization fronts as a signature of transient reconnection in the magnetotail, J. Geophys. Res., 114, A04202, doi:10.1029/2008JA013980, 2009.

Slavin, J. A., Lepping, R. P., Gjerloev, J., Fairfield, D. H., Hesse, M., Owen, C. J., Moldwin, M. B., Nagai, T., Ieda, A., and Mukai, T.: Geotail observations of magnetic flux ropes in the plasma sheet, J. Geophys. Res., 108, A11015, doi:10.1029/2002JA009557, 2003a.

Slavin, J. A., Lepping, R. P., Gjerloev, J., Goldstein, M. L., Fairfield, D. H., Acuna, M. H., Balogh, A., Dunlop, M., Kivelson, M. G., Khurana, K., Fazakerley, A., Owen, C. J., Reme, H., and Bosqued, J. M.: Cluster electric current density measurements within a magnetic flux rope in the plasma sheet, Geophys. Res. Lett., 30, 1362, doi:10.1029/2002GL016411, 2003 b.

Sonnerup, B. U. Ö., Hasegawa, H., Teh, W.-L., and Hau, L.-N.: Grad-Shafranov reconstruction: An overview, J. Geophys. Res., 111, A09204, doi:10.1029/2006JA011717, 2006.

Sormakov, D. A. and Sergeev, V. A.: Topology of magnetic flux ropes in the magnetospheric plasma sheet as measured by the Geotail spacecraft, Cosmic Res., 46, 387-391, 2008.

Speiser, T. W.: Particle trajectories in model current sheets, J. Geophys. Res., 70, 4219-4226, 1965.

Speiser, T. W.: Particle motion in the tail current sheet, Adv. Space Res., 11, 151-159, 1991.

Teh, W.-L., Nakamura, R., Karimabadi, H., Baumjohann, W., and Zhang, T. L.: Correlation of core field polarity of magnetotail flux ropes with the IMF $B_{y}$ : Reconnection guide field dependency, J. Geophys. Res., 119, 2933-2944, doi:10.1002/2013JA019454, 2014.

Treumann, R. A., Baumjohann, W., and Gonzalez, W. D.: Collisionless reconnection: magnetic field line interaction, Ann. Geophys., 30, 1515-1528, doi:10.5194/angeo-30-1515-2012, 2012.

Vogiatzis, I. I., Fritz, T. A., Zong, Q.-G., Baker, D. N., Sarris, E. T., and Daly, P. W.: Fine-time energetic electron behavior observed by Cluster/RAPID in the magnetotail associated with X-line formation and subsequent current disruption, Ann. Geophys., 23, 2265-2280, doi:10.5194/angeo-23-2265-2005, 2005.

Vogiatzis, I. I., Malandraki, O. E., Zong, Q.-G., Zhou, X.-Z., Sarris, T. E., Sarris, E. T., Zhang, H., and Fritz, T. A.: THEMIS observations of earthward convected flux ropes triggering field dipolarization/substorm expansion and associated particle ener- 
gization, Ann. Geophys., 29, 2117-2130, doi:10.5194/angeo-292117-2011, 2011.

Walsh, A. P., Fazakerley, A. N., Lahiff, A. D., Volwerk, M., Grocott, A., Dunlop, M. W., Lui, A. T. Y., Kistler, L. M., Lester, M., Mouikis, C., Pu, Z., Shen, C., Shi, J., Taylor, M. G. G. T., Lucek, E., Zhang, T. L., and Dandouras, I.: Cluster and Double Star multipoint observations of a plasma bubble, Ann. Geophys., 27, 725-743, doi:10.5194/angeo-27-725-2009, 2009.
Yao, Z. H., Pu, Z. Y., Fu, S. Y., Angelopoulos, V., Kubyshkina, M., Xing, X., Lyons, L., Nishimura, Y., Xie, L., Wang, X. G., Xiao, C. J., Cao, X., Liu, J., Zhang, H., Nowada, M., Zong, Q.-G., Guo, R. L., Zhong, J., and Li, J. X.: Mechanism of substorm current wedge formation: THEMIS observations, Geophys. Res. Lett., 39, L13102, doi:10.1029/2012GL052055, 2012. 